\title{
Resource scheduling algorithm optimization for multitarget inverse synthetic aperture radar imaging in radar network
}

\author{
Helei Wang $\odot$, ${ }^{\text {a }}$ Kefei Liao $\odot,{ }^{\text {a,b,* }}$ Shan Ouyang, ${ }^{\text {a,b }}$ Haitao Wang, ${ }^{\text {a }}$ \\ and Liping Yang ${ }^{\mathbf{a}}$ \\ ${ }^{a}$ Guilin University of Electronic Technology, College of Information and Communication, \\ Guilin, China \\ ${ }^{\mathrm{b}}$ Guilin University of Electronic Technology, State and Local Joint Engineering Research Center \\ for Satellite Navigation and Location Service, Guilin, China
}

\begin{abstract}
Aiming at optimizing the allocation problem of limited resources in a radar network, a resource scheduling algorithm combining pulse interleaving with preallocation is proposed for multitarget inverse synthetic aperture radar imaging. The imaging method adopts compressed sensing, which only needs to emit a small number of pulses so that we can set the algorithms to schedule the allocation of the pulses over a period of time. The authors point out the problem of pulse conflict, which is ignored in the process of the scheduling algorithm and proposes a preallocation method to avoid the occurrence of the conflict. Meanwhile, pulse interleaving is added to increase the positions of the dispatchable pulses. Moreover, the combined algorithm can perform adaptive scheduling on the radar time resource according to the feature parameters after target feature cognitive. Finally, the feasibility of the combined algorithm is verified by the simulation, and two performance indicators, the hit value rate and the pulse utilization rate, are improved by the proposed algorithm. (C) The Authors. Published by SPIE under a Creative Commons Attribution 4.0 Unported License. Distribution or reproduction of this work in whole or in part requires full attribution of the original publication, including its DOI. [DOI: 10.1117/1.JRS.15.016521]
\end{abstract}

Keywords: ISAR imaging; resource scheduling; radar network; pulse interleaving; preallocation.

Paper 200861 received Dec. 7, 2020; accepted for publication Mar. 10, 2021; published online Mar. 31, 2021.

\section{Introduction}

With the complexity and diversity of radar application scenes and the increase in noncooperative targets in the air, it is difficult to obtain more comprehensive target information only relying on a single radar to image the target. Therefore, the application and rise of radar networks are imperative, and it also requires more tasks to be performed with limited radar resources. ${ }^{1-3}$ Adaptive scheduling is the most effective method among many resource scheduling methods. By introducing the idea of cognition and further improving the adaptive ability of radar network resource scheduling, the success rate of task scheduling can be greatly improved. ${ }^{4,5}$ Besides, compressed sensing (CS) plays a very important role in sparse inverse synthetic aperture radar (ISAR) imaging and saves considerable resources for other tasks. ${ }^{6-8}$

To date, some scholars have performed much research on resource scheduling. For example, a joint antenna layout and transmission power distribution algorithm was proposed to improve the monitoring performance of a distributed radar network in Ref. 9. Yan et al. ${ }^{10}$ proposed an optimal antenna deployment algorithm for the problem of multiregion simultaneous interference. Furthermore, Tian et al. ${ }^{11}$ proposed a time-constrained task scheduling algorithm for a multifunctional radar network task scheduling problem and verified the effectiveness of the algorithm. These studies all focus on resource scheduling for target detection, tracking, location, and target feature cognition.

*Address all correspondence to Kefei Liao, kefeiliao@guet.edu.cn 
At present, there are relatively few studies on the scheduling of imaging resources in radar networks. However, imaging needs to occupy most of the resources, so the reasonable allocation of imaging resources can save more resources for the radar to perform more tasks. ${ }^{12-14}$ The model established in Ref. 15 optimizes resource allocation for multiobjective imaging tasks in radar networks, but it uses the traditional Rang-Doppler (RD) imaging algorithm, which, although effective for traditional algorithm, is not as superior as CS. An allocation method of resource scheduling based on sparse observation ISAR imaging was proposed in Ref. 16, but it uses the allocation method based on pulse dwell time. On the other hand, Chen et al. introduced pulse interleaving technique to break through the original resource saturation upper limit that makes full use of the waiting time between transmitting and receiving pulses and maximizes the use of radar resources. ${ }^{17}$ However, both studies were carried out on a single radar.

A radar network is different from a single radar in that it is necessary to consider the conflict that arises from the same frequency pulse of multiple radars for a certain target. Based on the recognition of target characteristics, the proposed algorithm calculates the pulse number needed for the sparse observation of each target. Then, preallocation and pulse interleaving are used for adaptive allocation. Finally, the resource scheduling for multiple targets in the timing sequence of multiple radars is completed.

The rest of this paper is organized as follows. Section 2 introduces the concept of cognition and how to obtain cognitive parameters. Section 3 introduces the core technical support for this scheduling algorithm: preallocation and pulse interleaving. Furthermore, the imaging model of the radar network resource scheduling algorithm is established. Section 4 gives the experimental simulation results and analysis. Finally, Sec. 5 draws the conclusions.

\section{Cognition of Target Characteristics}

Through learning from the surrounding environment, combining with prior information and reasoning, cognitive radar constantly adjusts the transmitter to realize feedback between receiver and transmitter so as to improve the overall performance of radars.

After receiving the echo signal, the cognitive radar network can calculate the specific information of the detected target using the conventional tracking algorithm of the traditional radar: the range $R_{i, j}$, the velocity $V_{i, j}$, and the angle $\Delta \theta_{i, j}$ that between the target and the center of the radar beam of the $i$ 'th radar to the $j$ 'th target. The other parameters according to Ref. 16 are calculated as follows:

(1) Priority $P_{i, j}$ : According to the priority criteria: it is generally believed that fast, close, radaroriented targets have a higher degree of threat for radar. Therefore, the priority of the threat degree of the $i$ 'th radar to the $j$ 'th target can be weighted by the three characteristic parameters of the range, velocity, and heading angle, and the equation is as follows:

$$
\begin{gathered}
P_{i, j}=\omega_{a} \frac{1 / R_{i, j}}{\max \left(1 / R_{i, j}\right)}+\omega_{b} \frac{V_{j}}{\max \left(V_{j}\right)}+\omega_{c}\left(1-\sin \Delta \theta_{i, j}\right), \\
1 \geq \omega_{a}, \omega_{b}, \omega_{c} \geq 0 \quad \text { and } \quad \omega_{a}+\omega_{b}+\omega_{c}=1 .
\end{gathered}
$$

Therefore, $P_{i, j} \in(0,1)$, and $w_{a}, w_{b}, w_{c}$ represent the weight coefficients of the parameters respectively.

(2) Size $S_{i, j}$ : The coarse-resolution image, $S_{i, j}^{k}$, of the $i$ 'th radar to the $j$ 'th target in the $k$ axis can be obtained after processing the echo signal received by each radar. The two dependent variables $f$ and $f_{\tau m}$ are the frequencies in fast time and slow time, respectively. The normalization of the ISAR image can be written as

$$
S_{i, j}^{k}\left(f, f_{\tau m}\right)=\frac{\left|S_{i, j}^{k}\left(f, f_{\tau m}\right)\right|-\max _{f, f_{\tau m}}\left|S_{i, j}^{k}\left(f, f_{\tau m}\right)\right|}{\max _{f, f_{\tau m}}\left|S_{i, j}^{k}\left(f, f_{\tau m}\right)\right|-\min _{f, f_{\tau m}}\left|S_{i, j}^{k}\left(f, f_{\tau m}\right)\right|} .
$$


Because the size of target $S_{i, j}$ is directly related to the quality of radar imaging, the target size is obtained by the product of the range-oriented size, $S_{i, j}^{y}$, and the azimuth-oriented size, $S_{i, j}^{x}$. The target size can be obtained through ISAR imaging normalization analysis, and the equation is as follows:

$$
\left\{\begin{array}{l}
S_{i, j}^{x}=\left(f_{\text {big__tm }(i, j)}-f_{\text {small_zm(i,j) }}\right) T_{c} \rho_{c} \\
S_{i, j}^{y}=\left(f_{\text {big }(i, j)}-f_{\text {small }(i, j)}\right) T_{p} \rho_{r} \\
S_{i, j}=S_{i, j}^{x} S_{i, j}^{y}
\end{array} .\right.
$$

$f_{\text {big_} \_m(i, j)}$ and $f_{\text {small_ } \_m(i, j)}$ represent the maximum and minimum frequencies, respectively, in the slow time direction of $S_{i, j}^{k} ; T_{c}$ represents the coherent accumulation time required to image the target; $\rho_{c}$ represents the azimuthal resolution in the target imaging, and it depends on bandwidth $B ; f_{\operatorname{big}(i, j)}$ and $f_{\text {small }(i, j)}$ represent the maximum and minimum frequencies in the fast time direction of $S_{i, j}^{k} ; T_{p}$ stands for the pulse width; and $\rho_{r}$ represents the range resolution in the target imaging. $S_{i, j}$ represents the estimated two-dimensional (2D) size of the final target.

It should be noted that in the radar network, the fusion of target information should be three-dimensional (3D) imaging. The image formed by the abovementioned single radar on the target is 2D, but the 3D projection model can be seen in Refs. 18 and 19. From the perspective of the 3D size information, the 3D size of the target is calculated by projection fusion, which is similar to the calculation principle of 2D. Because the research focus of this paper is the resource scheduling of cognitive radar networks, the following sections only carry out simple 3D projection fusion processing for target-size information.

(3) Azimuthal Coherent Accumulation Time $T_{i, j}^{c}$ : The coherent accumulation time of the $i$ 'th radar to the $j$ 'th target is determined as

$$
T_{i, j}^{c}=\frac{S_{\mathrm{ref}(i, j)}^{x}}{S_{i, j}^{x}} \cdot \frac{R_{i, j}}{V_{i, j}\left|\cos \left(\Delta \theta_{i, j}\right)\right|} \cdot \frac{\lambda}{2 \rho_{\mathrm{ref}}},
$$

where $\rho_{\text {ref }}$ is the desired azimuthal resolution of the target with datum azimuthal size of $S_{\operatorname{ref}(i, j)}^{x}$.

(4) Dimension Observed in Azimuth Direction $D_{i, j}: D_{i, j}$ represents the observation dimension of the $j$ 'th target in the $i$ 'th radar, which is represented by the number of pulses in time. According to CS theory, only when the equation of observation dimension is satisfied can the reconstruction algorithm reconstruct the original signal with high probability. The equation can be written as

$$
D_{i, j} \geq c_{1} K_{i, j} \ln \left(f_{\mathrm{PRF}} \cdot T_{i, j}^{c}\right),
$$

where $c_{1}$ is a small constant between 0.5 and 2 , and $f_{\mathrm{PRF}}$ is the pulse repetition frequency of the transmitted radar signal. For $K_{i, j}$, it is the sparsity of Doppler-domain echo in the $i^{\prime}$ th target in the $j$ 'th radar, which is determined by the coarse resolution in Eq. (3). Setting a pro per threshold $E_{i, j}$ and letting vector $\overrightarrow{S_{i, j}^{k}}$ be the discrete representation of $S_{i, j}^{k}, K_{i, j}$ is defined as the number of elements that are greater than $E_{i, j}$ in $\overrightarrow{S_{i, j}^{k}}$.

\section{Scheduling Algorithm}

\subsection{Preallocation}

When sparse ISAR uses CS to reconstruct image information, it is easy to see the phenomenon shown in Fig. 1, where multiple radars image a target. It shows that the simultaneous pulse transmission of multiple radars to the target at some time causes mutual interference among the radars and affects the echo signal. To be more specific, each radar is only eager to receive its own signals. When multiple radars transmit the same frequency signal at the same time, the 


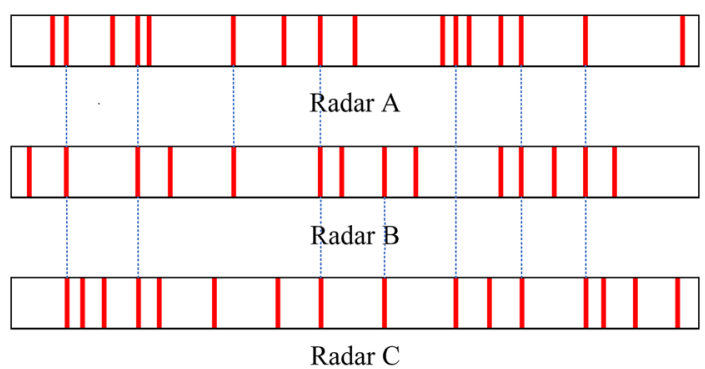

Fig. 1 Pulse conflict.

receiver cannot judge which signal is transmitted by the same radar transmitter, thus causing interference. Consequently, if this situation is not avoided, it will not only waste the resources but also will affect the imaging effect when the radar resources are not adequately allocated.

Therefore, the transit scheduling interval can be used to preallocate each target, to avoid this kind of conflict. The specific method is shown in Fig. 2, where $T_{j}$ represents the virtual scheduling interval of the $j$ 'th target. Considering three radars imaging the same target, the detailed steps of preallocation are described below.

Step 1: First, every radar will remove the observations of some target with lower priority. Then, for one target appearing in multiple radars' allocation objects, three radars with higher priority, A, B, and C, will be selected and sorted.

Step 2: According to the order obtained in step 1, the free positions in the transit scheduling interval are randomly assigned to the three radars in turn, and the radar marks are made. Step 3: Transfer the assigned results to the corresponding radar timing sequence to avoid conflicts.

Because of the sparsity, it is possible to allocate several radars in one transit scheduling interval without conflict. However, if only the pulse conflict is reduced, the conflict is not serious when the number of targets is small. And less spare time can be allocated when the number of targets is large, but it can prevent low-priority tasks from overwriting high-priority tasks because they are allocated in order of priority.

Hence in both cases, the improvements of resource utilization rate and scheduling success rate (SSR) are not very great in the radar network. Furthermore, this theory is verified by simulation in Sec. 4 of this paper. Therefore, pulse interleaving is proposed to provide more resources to be allocated when there are more targets. Although Ref. 17 completed the application of pulse interleaving for a single radar, research on networking has not been further studied.

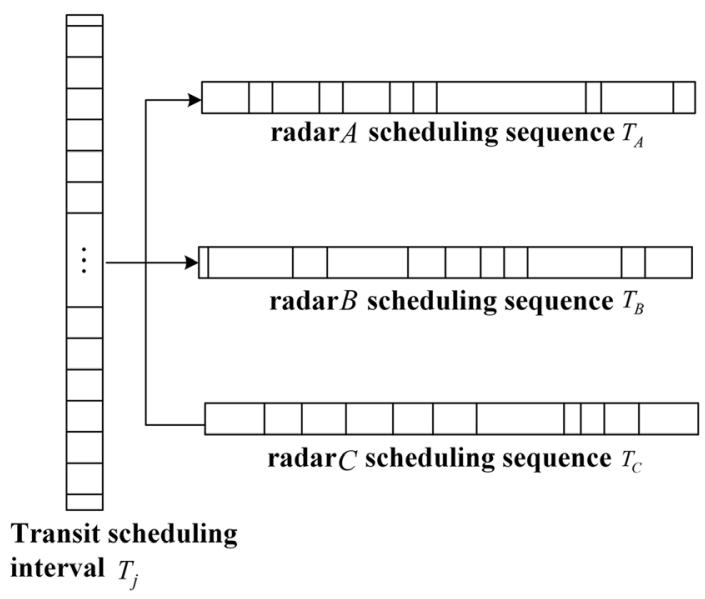

Fig. 2 Schematic diagram of preallocation. 


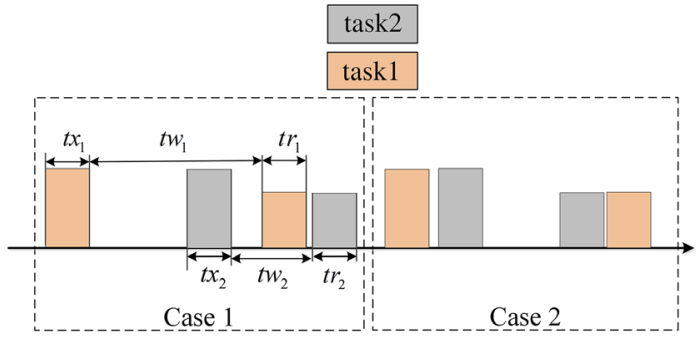

Fig. 3 Pulse interleaving.

\subsection{Pulse Interleaving}

The dwell time of the radar task includes three processes: pulse transmitting, waiting, and receiving. Most studies regard the three parts as a whole, which wastes the intermediate waiting time. Pulse interleaving is used to insert other tasks into the waiting time to make full use of the resources, and it can be expressed in Fig. 3.

The two interleaving cases of pulse interleaving were introduced, corresponding to the left and right cases in Fig. 3, respectively. And the time constraints of the two cases are as follows:

$$
\begin{gathered}
\left\{\begin{array}{l}
t w_{1} \geq t x_{2}, \\
t w_{2} \geq t r_{1}
\end{array}\right. \\
t w_{1} \geq t x_{2}+t w_{2}+t r_{2},
\end{gathered}
$$

where $t x$ is the length of time to transmit the pulse, $t w$ is waiting time for between the transmitting and receiving pulses, and $t r$ is the length of time to receive the pulse. As long as there is no conflict between the pulses no matter what the case is adopted, and the spare time is fully used, the utilization rate of pulse resources can be improved.

\subsection{Algorithm Mode}

Since there are many variable factors, we can define several positive indexes of the scheduling algorithm and construct the objective function from these performance indicators so that we can obtain the optimal solution by finding the maximum value of the objective function under some constraints in the algorithm model. Therefore, two resource scheduling performance indicators can be defined.

(1) Hit value rate: The ratio of the sum of the actual completing task priorities to the sum of the requested task priorities. Different from the ratio of the implemented tasks to the total tasks (ROI), ${ }^{16}$ each task is multiplied by its corresponding priority in the way the hit value rate (HVR) is calculated, which makes the HVR more representative. In Eq. (9), $X_{i, j}$ is an element in a selection matrix, indicating whether the $j$ 'th target is allocated in the $i$ 'th radar; $N_{s}$ and $N$ are the number of imaging targets actually executed and the number of imaging targets applied for execution, respectively:

$$
\mathrm{HVR}=\frac{\sum_{i=1}^{M} \sum_{j=1}^{N_{s}}\left(X_{i, j} P_{i, j}\right)}{\sum_{i=1}^{M} \sum_{j=1}^{N}\left(X_{i, j} P_{i, j}\right)} .
$$

(2) Pulse utilization rate: The ratio of the number of pulses required to dispatch all mission targets to the total pulse of the radar network system. The value of PUR represents the resource utilization of the entire radar network system, which has the same meaning as time utilization rate (TUR) in Ref. 16. However, the representation method of TUR is too complicated, so it is changed to PUR to represent the performance of the algorithms. In Eq. (10), $T_{M}$ represents the total dispatching interval time for all radars: 


$$
\mathrm{PUR}=\frac{\sum_{i=1}^{M} \sum_{j=1}^{N}\left(X_{i, j} D_{i, j}\right)}{T_{M} f_{\mathrm{PRF}}}
$$

Suppose there are $M$ radars and $N$ targets, and each target is observed simultaneously by three radars in the radar network. Through the above analysis, the model of the prescheduling algorithm for radar network resources based on pulse interleave can be built as follows:

$$
\begin{gathered}
\max _{X_{i, j} P_{i, j}}\left(Q_{1} \frac{\sum_{i=1}^{M} \sum_{j=1}^{N_{s}}\left(X_{i, j} P_{i, j}\right)}{\sum_{i=1}^{M} \sum_{j=1}^{N}\left(X_{i, j} P_{i, j}\right)}+Q_{2} \frac{\sum_{i=1}^{M} \sum_{j=1}^{N}\left(X_{i, j} D_{i, j}\right)}{T_{M} f_{\mathrm{PRF}}}\right) \\
\text { s.t }\left\{\begin{array}{l}
1 X_{i, j} \in\{0,1\} \\
2 \forall a, b \in[1, M], \text { if }\left(X_{a, j} P_{a, j}>X_{b, j} P_{b, j}\right) ;\left(X_{a, j} t_{a, j}<X_{b, j} t_{b, j}\right) \\
3 \forall j \in[1, N], \sum_{i=1}^{M} X_{i, j}=3 \text { (Three radars are not collinear) } \\
40 \leq t_{i, j} \leq t_{\mathrm{end}}-T_{i, j}^{c} \\
5 t w_{i, j}=\frac{2 R_{i, j}}{c} \\
6 \forall T_{i}, T_{j}, \bigcap_{j=1}^{N}\left\{[ t _ { i , j } ; t _ { i , j } + t x _ { i , j } ] \cup \left[t_{i, j}+t x_{i, j}\right.\right. \\
\left.7 w_{i, j}: t_{i, j}+t x_{i, j}+t w_{i, j}+t r_{i, j}\right]
\end{array}\right\}=\varnothing \\
8 \sum_{i, j} \geq c_{1} K_{i, j} \ln \left(f_{\mathrm{PRF}} \cdot T_{i, j}^{c}\right)
\end{gathered} .
$$

$Q_{1}$ and $Q_{2}$ are the adjustment coefficients in the objective function, and we assume $Q_{1}=Q_{2}=0.5$. The first constraint represents the value range of $X_{i, j}$. The second constraint indicates that the radar with high priority under the same target should allocate resources first, and $t_{i, j}$ is the initial observation time of the $j$ 'th target in the $i^{\prime}$ th radar. To simplify the analysis, the third constraint indicates that each target in this paper will be observed by three noncollinear radars. In the fourth constraint, $t_{\text {end }}$ is the set observation cutoff time so this constraint represents the range of the initial observation time. In the fifth constraint, the waiting time of different radars for different targets is different, which is determined by the distance between radar and target. The sixth constraint refers to the situation in which resources are not preempted in the radar timing sequence and transit scheduling interval timing sequence, where $T_{i}$ represent the radar timing sequence of the $i$ 'th radar. The seventh constraint is the condition of target sparse reconstruction, which is the precondition of imaging quality. In the eighth constraint, $T$ is the total resources of the radar network system, and $N_{s i}$ is the number of targets to be dispatched in the $i$ 'th radar; then, this equation represents that the total resources used in dispatching are less than $T$.

The above are the constraints of the combined algorithm in the radar network that we proposed. After applying these constraints, the random time sequence results of each subradar of the radar network with multiple targets can be obtained. Although a genetic algorithm can be used to obtain the optimal solution, ${ }^{20}$ considering the randomness of CS, the solution was obtained directly under the constraint condition of completion. In addition, the idea of greedy algorithm $^{21}$ is used to allocate as many resources as possible when using preallocation. Therefore, the final solution steps are as follows:

Step 1: A small number of pulses are emitted to recognize the target characteristics. Then according to the echo signal and the radar corresponding to each target determined by the priority, the azimuthal coherent accumulation time $T_{i, j}^{c}$ and observation dimension $D_{i, j}$ required for each target image are calculated.

Step 2: A target is distributed among multiple radars by the preallocation, and pulse conflicts among multiple radars are avoided in that way.

Step 3: The allocation result of step 2 is transferred to the corresponding radar timing sequence. However, since a single radar will transmit different pulses to multiple targets. It may happen that some locations have been occupied by high-priority targets in the process of 


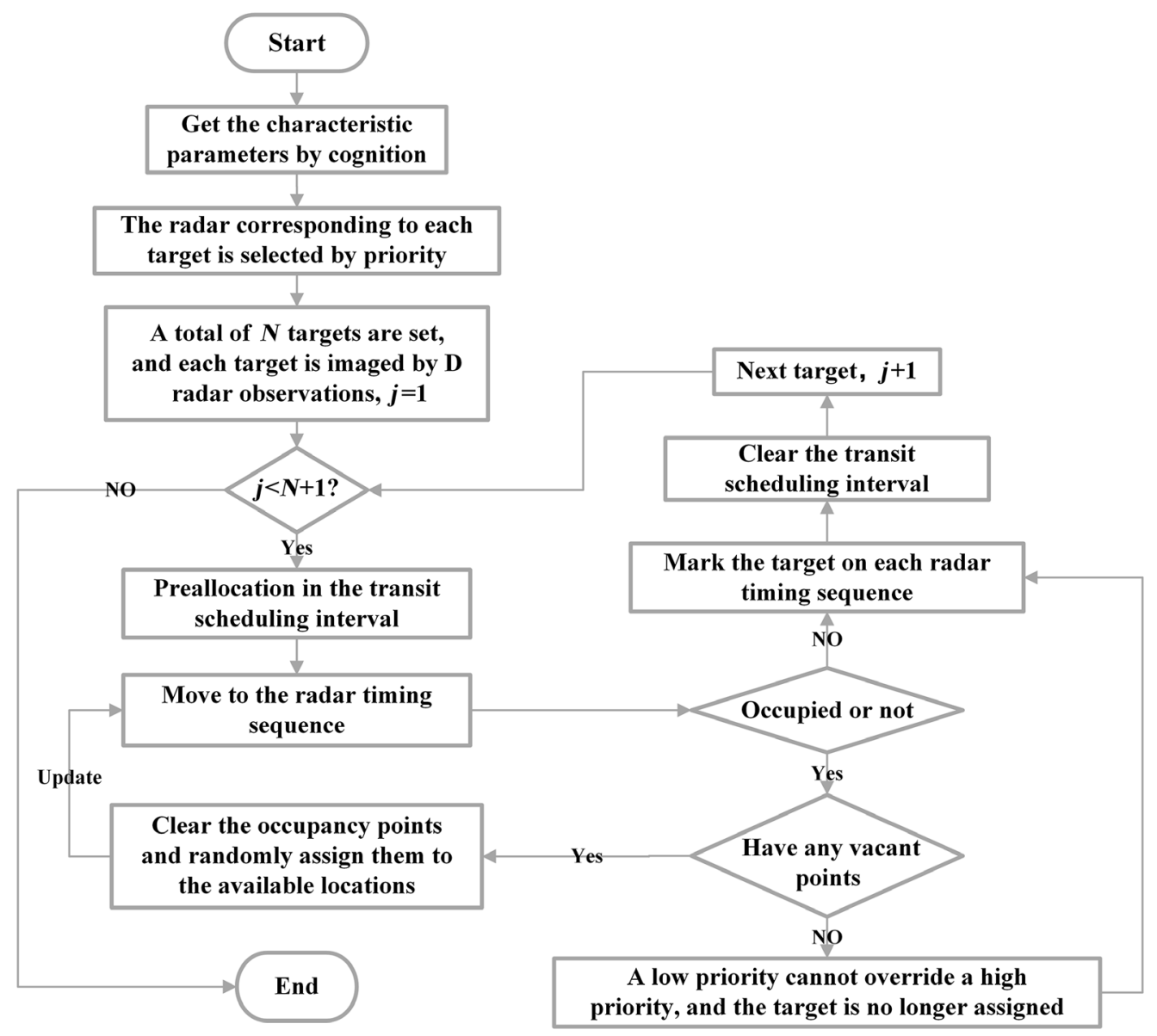

Fig. 4 Algorithm process.

moving to radar timing when a target is allocated timing sequences without pulse conflict. Skip step 4 if there is no occupancy.

Step 4: When an occupied position is found, it is necessary to check if there are any vacant positions. If so, the occupied position is cleared in the transit scheduling interval and randomly allocated in a remaining available position.

Step 5: After the transit scheduling interval is successfully transferred to the corresponding radar timing sequence, the target is transferred on the mark, then the transit scheduling interval is cleared, and the process returns to step 2 to perform the same operation on the next target until all targets are scheduled.

The algorithm process of radar resource scheduling based on pulse interleaving is shown in Fig. 4.

\section{Simulation Results}

Simulation experiments are performed to verify the validity and superiority of the proposed task allocation and scheduling algorithm for a radar network. It is assumed that the system of the radar network is composed of six noncollinear ISARs on the ground, which are denoted by $i(i=1,2, \ldots, 6)$. There are eight targets that need to be imaged in the air, which are represented by $j(j=1,2, \ldots, 8)$. Assume that all radars emit chirp signals, the carrier bandwidth $B=300 \mathrm{MHz}$, the carrier frequency $f_{c}=10 \mathrm{GHz}$, the pulse width $T_{p}=1 \mu \mathrm{s}$, and the pulse repetition frequency $f_{\mathrm{PRF}}=1000 \mathrm{~Hz}$. To ensure a sufficient scheduling interval in the case of multiple targets, it is assumed that the number of reconstructed observation dimension for each radar is 10,000 ( $1 \mathrm{~s}$ of observation time). 
Wang et al.: Resource scheduling algorithm optimization for multitarget inverse synthetic aperture radar...

First, the set scene is used to obtain the coarse resolution image of the target according to the traditional ISAR imaging method. Then, the values of various characteristics of the target are calculated by the equations in Sec. 2. The parameters of the observation dimension, coherent accumulation time, and distance are shown in Tables $1-3$, respectively (all three tables are the result of the product with the radar selection matrix).

Table 1 The coherent accumulation time of each target in each radar $(0.1 \mathrm{~ms})$.

\begin{tabular}{lcccccc}
\hline \hline & Radar1 & Radar2 & Radar3 & Radar4 & Radar5 & Radar6 \\
\hline Target1 & 0 & 8605 & 9030 & 0 & 0 & 9480 \\
Target2 & 0 & 0 & 9079 & 9330 & 9530 & 0 \\
Target3 & 9325 & 0 & 0 & 8681 & 0 & 8805 \\
Target4 & 0 & 8878 & 9463 & 0 & 9310 & 0 \\
Target5 & 9466 & 0 & 0 & 0 & 8967 & 9481 \\
Target6 & 8867 & 9516 & 0 & 8948 & 0 & 0 \\
Target7 & 0 & 0 & 8792 & 0 & 8893 & 8694 \\
Target8 & 9561 & 8967 & 0 & 8762 & 0 & 0 \\
\hline \hline
\end{tabular}

Table 2 The observation dimensions of each target corresponding to each radar.

\begin{tabular}{lcccccc}
\hline \hline & Radar1 & Radar2 & Radar3 & Radar4 & Radar5 & Radar6 \\
\hline Target1 & 0 & 830 & 767 & 0 & 0 & 704 \\
Target2 & 0 & 0 & 692 & 743 & 751 & 0 \\
Target3 & 762 & 0 & 0 & 768 & 0 & 756 \\
Target4 & 0 & 753 & 766 & 0 & 740 & 0 \\
Target5 & 711 & 0 & 0 & 0 & 670 & 750 \\
Target6 & 721 & 729 & 0 & 750 & 0 & 0 \\
Target7 & 0 & 0 & 750 & 0 & 738 & 747 \\
Target8 & 751 & 746 & 0 & 732 & 0 & 0 \\
\hline \hline
\end{tabular}

Table 3 Distance between each target and each radar $(\mathrm{km})$.

\begin{tabular}{lcccccc}
\hline \hline & Radar1 & Radar2 & Radar3 & Radar4 & Radar5 & Radar6 \\
\hline Target1 & 0 & 9 & 10 & 0 & 0 & 11 \\
Target2 & 0 & 0 & 11 & 10 & 11 & 0 \\
Target3 & 12 & 0 & 0 & 10 & 0 & 10 \\
Target4 & 0 & 10 & 12 & 0 & 9 & 0 \\
Target5 & 10 & 0 & 0 & 0 & 12 & 13 \\
Target6 & 11 & 9 & 0 & 10 & 0 & 0 \\
Target7 & 0 & 0 & 11 & 0 & 13 & 11 \\
Target8 & 11 & 11 & 0 & 10 & 0 & 0 \\
\hline \hline
\end{tabular}



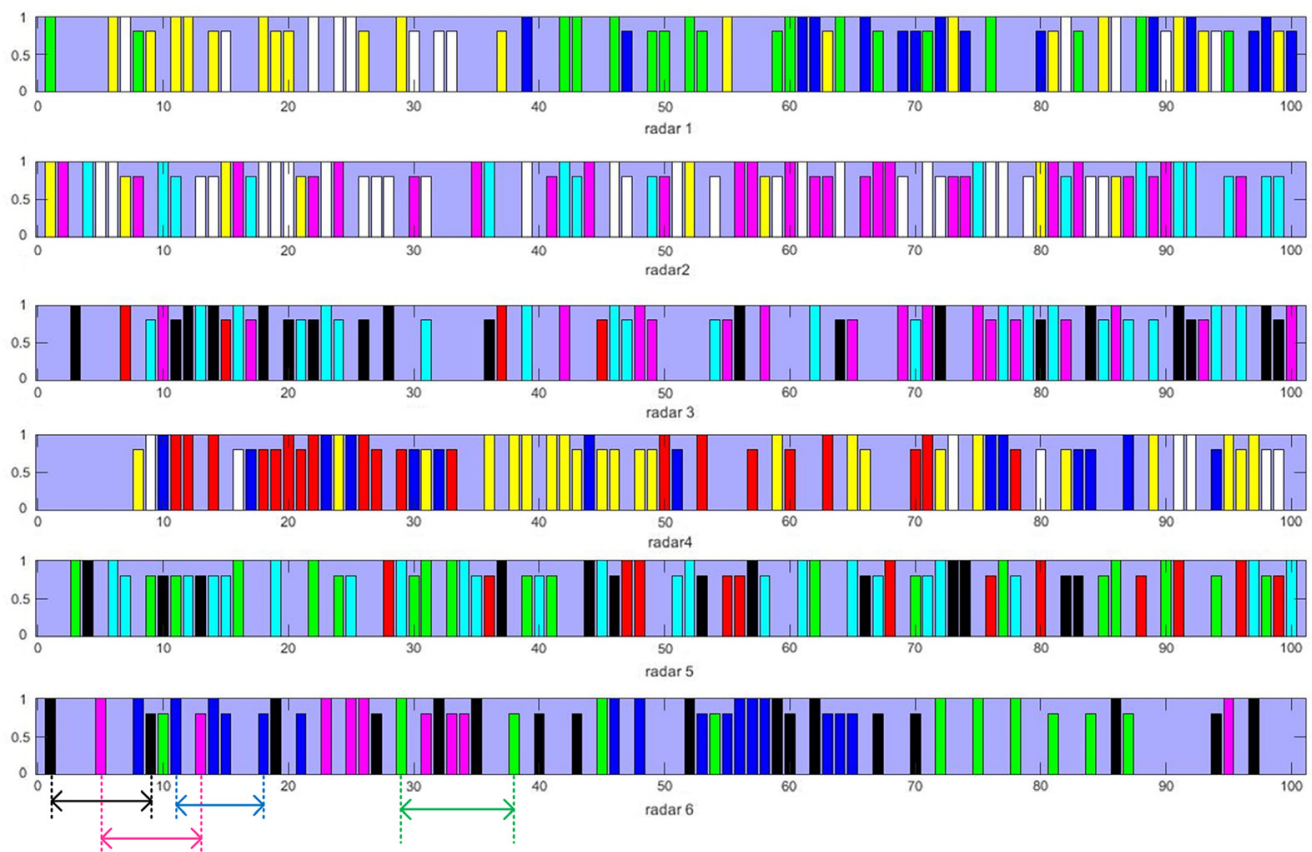

(a)

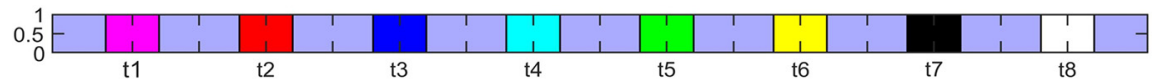

(b)

Fig. 5 Combined algorithm scheduling sequence. (a) The first 100 points and (b) the color of the targets.

According to the distances in Table 3, the dwell time is $\sim 10$ times that of a single transceiver pulse. If the dwell time of each pulse is taken as the minimum unit, the number of points in Table 1 will be $\sim 1 / 10$ of the original data, and the number of observation dimension in per radar is 1000. At this time, the number of observation dimension required by each radar in Table 2 is more than 2000, and the imaging of each target cannot be completed. Therefore, the use of pulse interleaving can break through the upper limit of the original resource saturation and improve the resource utilization rate.

Following the algorithm process introduced in Sec. 3, the pulse time sequence diagram of each radar can be obtained as shown in Fig. 5(a), which shows the distribution of the first 100 points intercepted. In this figure, arrows are marked under the time sequence diagram of radar 6 . The whole length of one arrow represents the dwell time, and the two ends are transmitting and receiving pulses. In general, it can be seen that more signals can be transmitted within the same period of time after using combined algorithm to make full use of resources.

For the convenience of observation in the above experimental simulation, the observation times of multiple targets are relatively concentrated, i.e., the observation start time is relatively close, which should be relatively dispersed in the actual situation so that the timing sequence of each radar is continuous. The allocation of observation time of multiple targets in the radar network can be referred to Refs. 15 and 22.

The two performance indicators, HVR and PUR, will be used to compare the differences of the proposed algorithm and the traditional algorithm. According to the calculation method of the two indicators, the results of the four algorithms under different target numbers are shown in Figs. 6 and 7, where the ratio is the average value after 50 Monte Carlo experiments.

In Figs. 6 and 7, the traditional algorithm is the algorithm that does not use the pulse interleaving technique and preallocation technique such as Ref. 18. It is only used to image sparse targets with CS without other improvements, which is easy to cause pulse conflicts and highpriority be covered. The preallocation algorithm is the algorithm that adds the preallocation technique to the traditional algorithm. Likewise, the interleaving algorithm is the algorithm that adds 
Wang et al.: Resource scheduling algorithm optimization for multitarget inverse synthetic aperture radar...

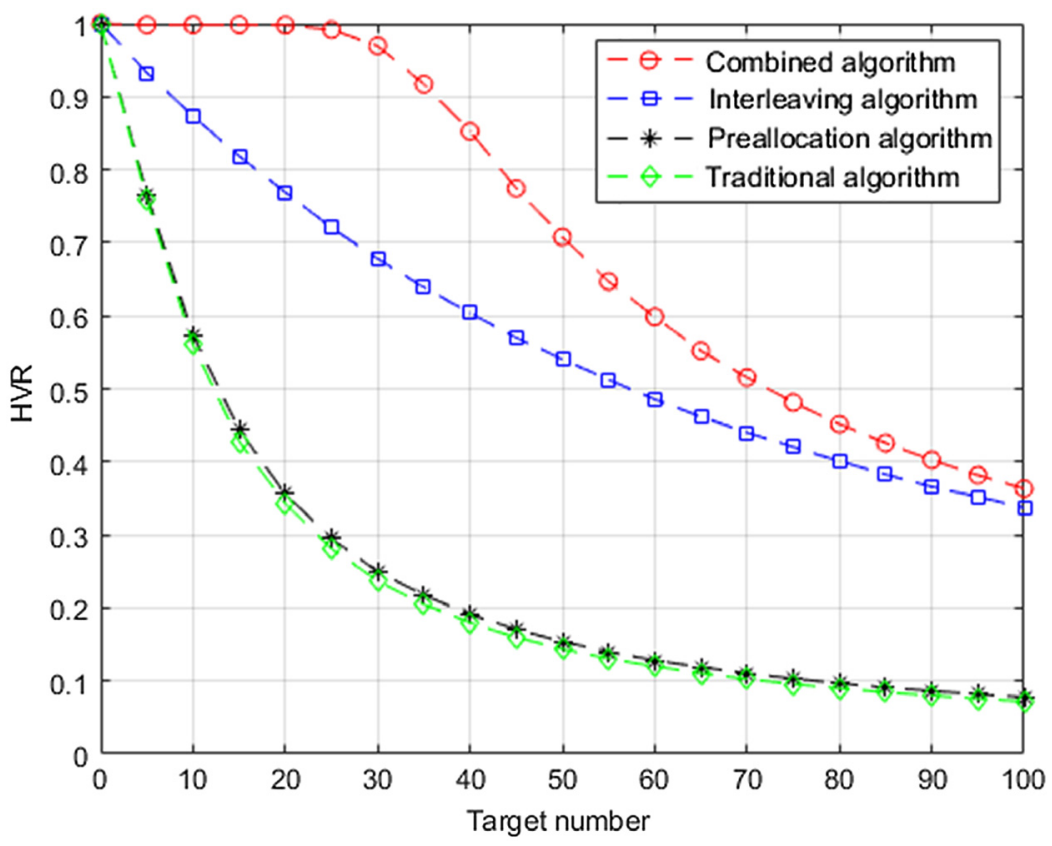

Fig. 6 Hit value ratio comparisons with the same priorities.

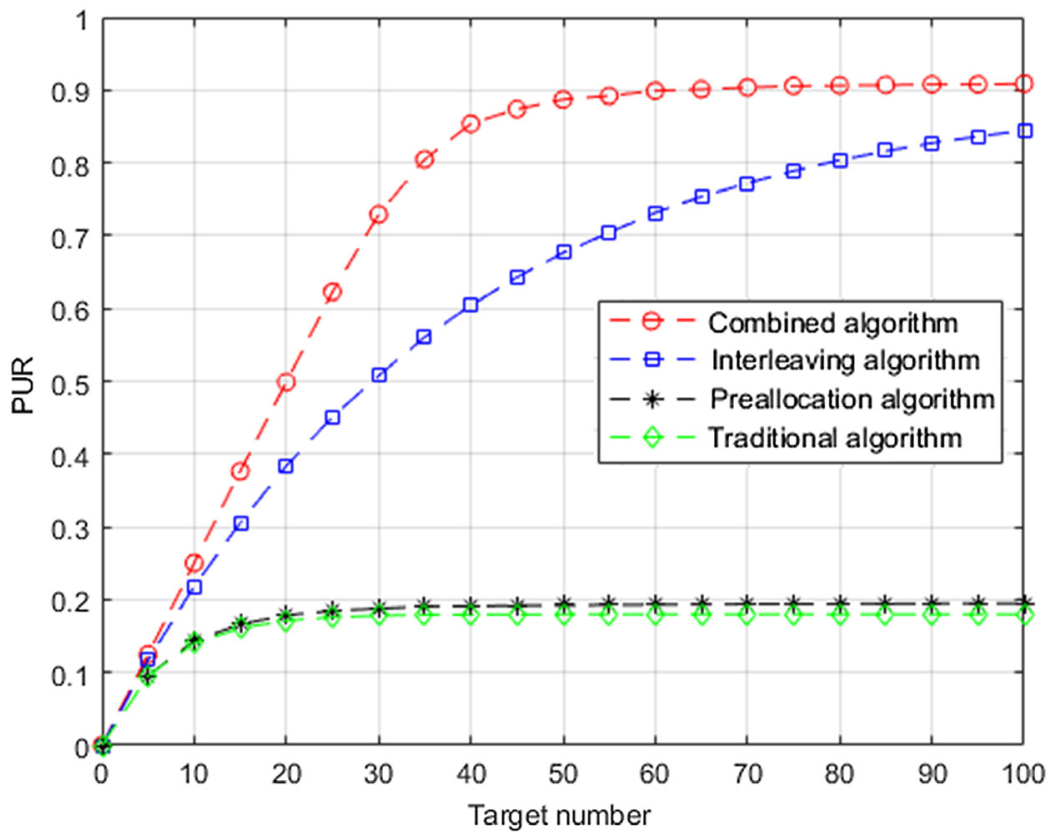

Fig. 7 Pulse utilization ratio comparisons.

the interleaving technique to the traditional algorithm. And the combined algorithm is the algorithm that uses the preallocation and pulse interleaving technique.

In terms of preallocation, compared with the traditional algorithm and preallocation algorithm, preallocation algorithm has less improvement on the basis of traditional algorithm. But compared with the combined algorithm and the interleaving algorithm, the effect of preallocation is amplified and combined with the interleaving algorithm well. In other words, the combination of preallocation technique and interleaving technique can enlarge the effect of each other. For pulse interleaving, its improvement is obvious because both the pulse interleaving algorithm and the combined algorithm using pulse interleaving technique are much higher value of PUR and HVR than the traditional algorithm and the pre-allocation algorithm without 
pulse interleaving. In the end, the combined algorithm is the best one whether it is in Fig. 6 or Fig. 7.

Compared with the traditional algorithm, the interleaving algorithm makes full use of the waiting time for pulse interleaving, whereas the traditional algorithm only occupies the transmitting and receiving pulse position of dwell time. Therefore, under the same resource, the utilization rate of the pulse resource is approximately $(t x+t w+t r) / 2$ times that of the traditional algorithm. This paper set up a dwell time used in the traditional unit that is 10 times that of a single pulse, so when finishing all the allocation of resources, the utilization rate of the pulse in the process of the interleaving algorithm can achieve five times that of the traditional algorithm. However, the waiting time of a target in a radar is fixed, and it needs to meet the requirements of transmitting and receiving pulses in a radar timing sequence simultaneously. Hence some separate spare positions cannot be used, and the final rate will be less than five times that of the traditional algorithm. It is $\sim 4.5$ times that of the traditional algorithm, which is in line with the result shown in Fig. 7.

Due to the random distribution of CS, the ratios of different target numbers in Figs. 6 and 7 vary slightly and are related to the set observation dimension, coherent accumulation time, radar observation number, and other parameters. Although there may be slight fluctuations at each point, the trends shown in the figures are consistent with the theoretical analysis of this component.

To visually observe the difference of the final imaging results, the scattered points of a target in the air are assumed as shown in Fig. 8, and the above four algorithms are used to image the air target as shown in Fig. 9.

It can be seen from Fig. 7 that the PUR of each algorithm is different, so within the same time length, the number of effective pulses that each algorithm can transmit is different. According to the data in Table 1-3, the simulation experiment found that the conflict points in the timing sequence were about $30 \%$ of the total resource points, and the echo signals of these conflict pulses could not be used as effective information. Therefore, if we assume that the combined algorithm can transmit 500 effective pulses over a period of time. If no preallocation technique is used, the interleaving algorithm can transmit 350 effective pulses. It can be seen from the above conclusion that the combined algorithm's pulse resource utilization ratio is about 4.5 times that of the traditional algorithm. Hence, the traditional algorithm can transmit 111 effective pulses, whereas the number of pulses that the preallocation algorithm can transmit is slightly larger than that of the traditional algorithm, which is set as 120 .

Through these data and CS techniques, simulation results of the four algorithms in Fig. 9 are obtained. It can be seen that within certain resources, with the improvement of the utilization rate

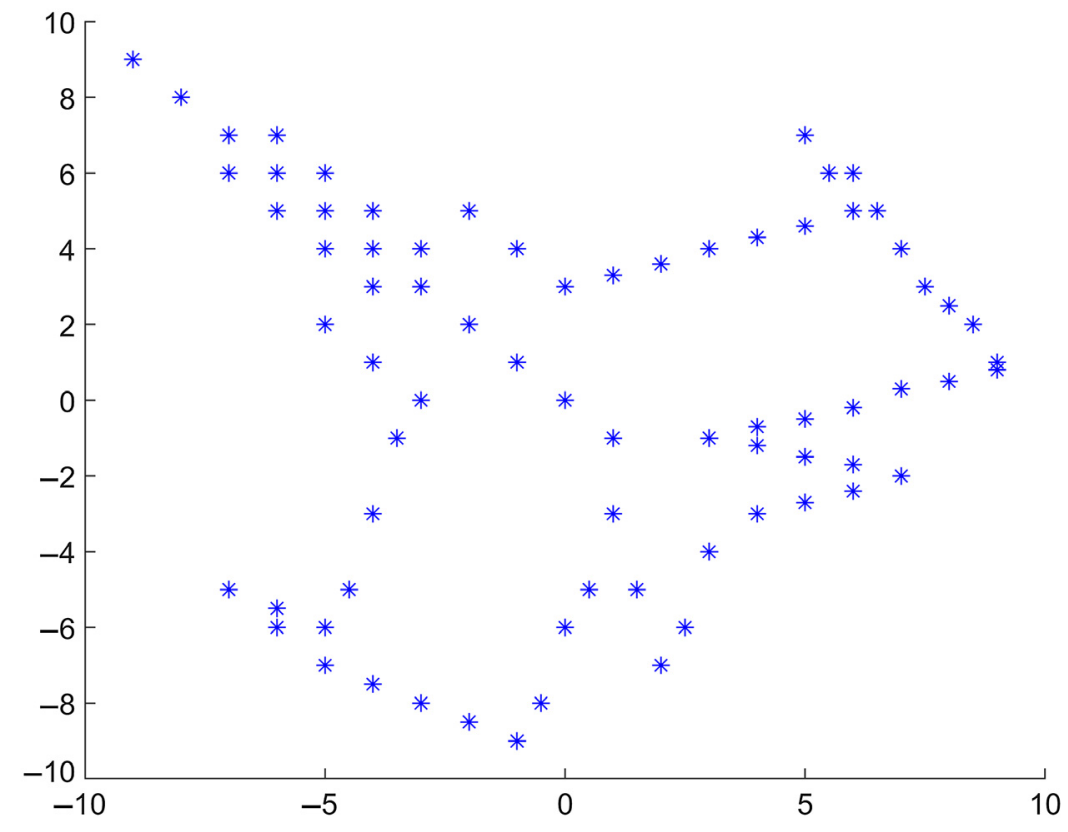

Fig. 8 Scattering points of the target. 


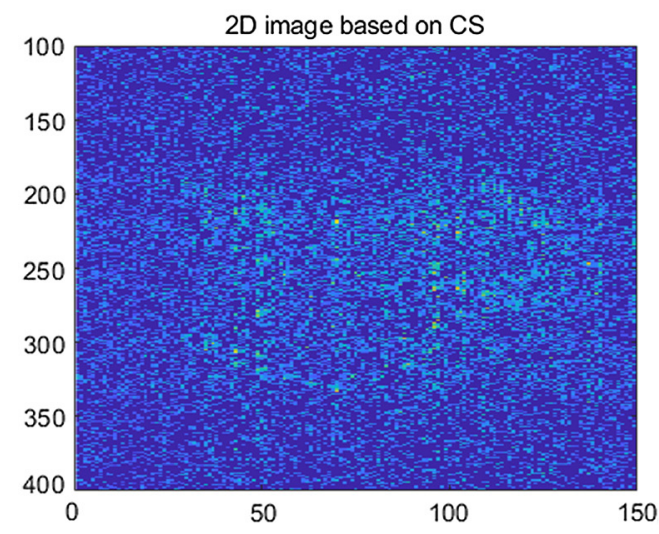

(a)

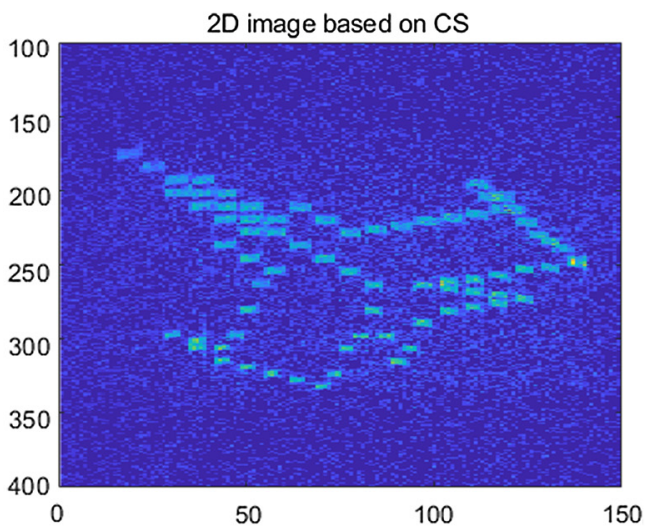

(c)

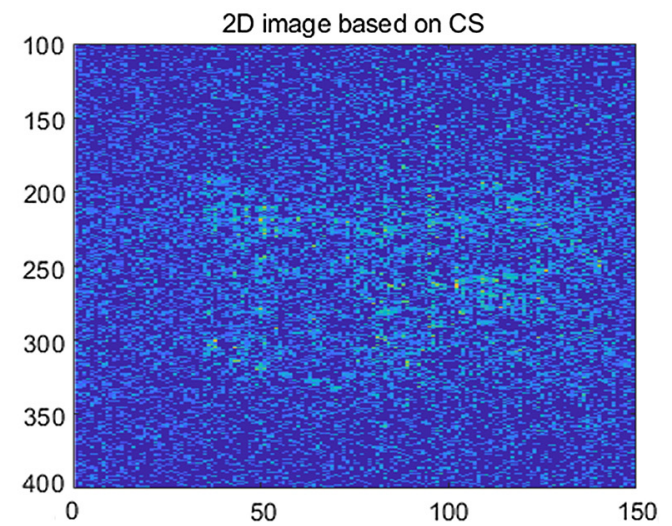

(b)

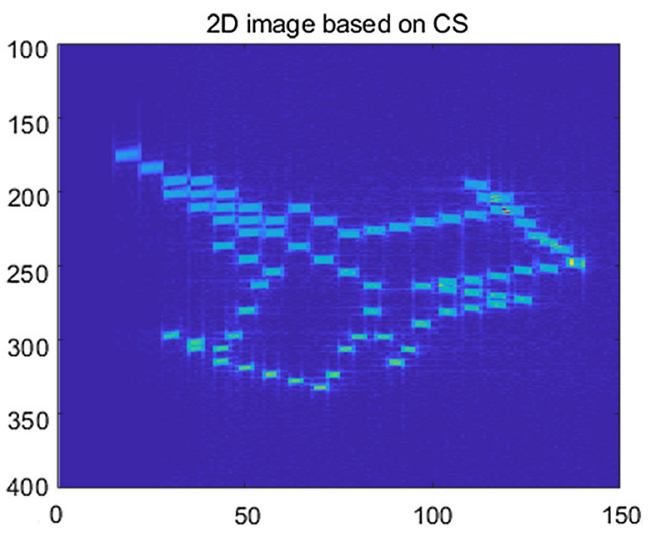

(d)

Fig. 9 Imaging comparisons. (a) Traditional algorithm with 111 effective pulses; (b) preallocation algorithm with 120 effective pulses; (c) interleaving algorithm with 350 effective pulses; and (d) combined algorithm with 500 effective pulses.

of pulse resources, the imaging effect is better. At the same time, it also reflects the great improvement of ISAR imaging in the combined algorithm proposed in this paper.

\section{Conclusion}

In this paper, we first studied the sparse ISAR imaging based on cognition and found that the problem of pulse conflict has been ignored when using CS imaging. Aiming at solving the problem, we proposed a method of preallocation that can be well combined with the pulse interleaving algorithm. The simulation results show that the combined algorithm can improve the pulse utilization ratio and hit value ratio so that the radar can perform more tasks. At the same time, the clearer image with the combined algorithm proves that it can complete the task with fewer resources. Moreover, we think it can be used not only for imaging of radar network but also for tracking and searching, and even for various multidimensional random scheduling problems. It should be noted that in this paper, we assume that the transmit pulse without energy constraint. But we take into account the time of receiving the pulse, which did not need too much energy. In our future work, we will continue to optimize the algorithm with more actual constraints and try to experiment in the actual scenario.

\section{Acknowledgments}

This work was supported by the National Natural Science Foundation of China (Grant Nos. 61631019, 61701128), Guangxi Science and Technology Department Project (AD18281061), and Postgraduate Innovation Program Project (2021YCXS038). 


\section{References}

1. T. A. Severson and D. A. Paley, "Distributed multitarget search and track assignment with consensus-based coordination," IEEE Sens. J. 15(2), 864-875 (2015).

2. Q. Liu et al., "Radar assignment for stealth targets detection and tracking based on BPSO in air-defense radar network," in IET Int. Radar Conf., pp. 1-5 (2013).

3. H. Griffiths, "Developments in bistatic and networked radar," in Proc. IEEE CIE Int. Conf. Radar, Chengdu, pp. 10-13 (2011).

4. K. L. Bell et al., "Cognitive radar framework for target detection and tracking," IEEE J. Sel. Top. Signal Process. 9(8), 1427-1439 (2015).

5. J. Yan et al., "Joint threshold adjustment and power allocation for cognitive target tracking in asynchronous radar network," IEEE Trans. Signal Process. 65(12), 3094-3106 (2017).

6. S. Zhang, Y. Liu, and X. Li, "Autofocusing for sparse aperture ISAR imaging based on joint constraint of sparsity and minimum entropy," IEEE J. Sel. Top. Appl. Earth Obs. Remote Sens. 10(3), 998-1011 (2017).

7. S. Zhang et al., "High-resolution bistatic ISAR imaging based on two-dimensional compressed sensing," IEEE Trans. Antennas Propag. 63(5), 2098-2111 (2015).

8. P. Cheng et al., "A fast and accurate compressed sensing reconstruction algorithm for ISAR imaging," IEEE Access 7, 157019-157026 (2019).

9. Y. Yang et al., "Multi-static radar power allocation for multi-stage stochastic task of missile interception," IET Radar Sonar Navig. 12(5), 540-548 (2018).

10. J. Yan et al., "Power allocation algorithm for target tracking in unmodulated continuous wave radar network," IEEE Sens. J. 15(2), 1098-1108 (2015).

11. T. Tian, T. Zhang, and L. Kong, "Timeliness constrained task scheduling for multifunction radar network," IEEE Sens. J. 19(2), 525-534 (2019).

12. X. Liu et al., "Game model in multi-target imaging task allocation," in Asia-Pacific Conf. Syn. Aperture Radar, pp. 1-5 (2019).

13. $\mathrm{S}$. Li et al., "ISAR imaging by two-dimensional convex optimization-based compressive sensing," IEEE Sens. J. 16(19), 7088-7093 (2016).

14. X. Liu et al., "Task allocation optimization for multi-target ISAR imaging in radar network," IEEE Sens. J. 18(1), 122-132 (2018).

15. D. Wang et al., "Task scheduling for multi-target ISAR imaging in radar network," IEEE Access 8, 46092-46101 (2020).

16. Y. Chen et al., "An adaptive ISAR-imaging-considered task scheduling algorithm for multi-function phased array radars," IEEE Trans. Signal Process. 63(19), 5096-5110 (2015).

17. J. Chen et al., "Multifunction phased radar resource management via maximal pulse interleaving technique," Arabian J. Sci. Eng. 38(11), 3081-3091 (2013).

18. L. Kang et al., "3D scattering image reconstruction based on measurement optimization of a radar network," Remote Sens. Lett. 11(7), 697-706 (2020).

19. Y. Du et al., "Time and aperture resource allocation strategy for multitarget ISAR imaging in a radar network," IEEE Sens. J. 20(6), 3196-3206 (2020).

20. L. Hao, X. Yang, and S. Hu, "Task scheduling of improved time shifting based on genetic algorithm for phased array radar," in IEEE Int. Conf. Signal Process., Chengdu, pp. 16551660 (2016).

21. L. Huang et al., "Task-scheduling scheme based on greedy algorithm in integrated radar and communication systems," J. Eng. 2019(19), 5864-5867 (2019).

22. Z. Qu, Z. Ding, and P. Moo, "A machine learning task selection method for radar resource management (poster)," in Int. Conf. Inf. Fusion, Ottawa, pp. 1-6 (2019).

Helei Wang is with the College of Information and Communication Guilin University of Electronic Technology, Guilin, China.

Kefei Liao is with the College of Information and Communication Guilin University of Electronic Technology, Guilin, China, also with State and Local Joint Engineering Research Center for Satellite Navigation and Location Service (Guilin University of Electronic Technology), Guilin, China. 
Wang et al.: Resource scheduling algorithm optimization for multitarget inverse synthetic aperture radar...

Shan Ouyang is with the College of Information and Communication Guilin University of Electronic Technology, Guilin, China, also with State and Local Joint Engineering Research Center for Satellite Navigation and Location Service (Guilin University of Electronic Technology), Guilin, China.

Haitao Wang is with the College of Information and Communication Guilin University of Electronic Technology, Guilin, China, also with State and Local Joint Engineering Research Center for Satellite Navigation and Location Service (Guilin University of Electronic Technology), Guilin, China.

Liping Yang is with the College of Information and Communication Guilin University of Electronic Technology, Guilin, China. 